\title{
Fetal Alcohol Spectrum Disorder service delivery: Exploring current systems of care from the caregivers' perspective
}

\author{
Lori Walls and Jacqueline Pei \\ Department of Educational Psychology, University of Alberta, Edmonton, AB, Canada
}

\begin{abstract}
Aims: The goals of this study were to: (1) gain improved understanding of the needs of caregivers caring for individuals with FASD; (2) gain improved understanding of the experiences of these caregivers as they attempt to access and implement supports in daily functioning; and (3) gain improved understanding of caregivers' perceptions of strengths and challenges in systems of care for individuals with FASD.

Design: Information for this study was gathered using a phenomenological in-depth interviewing method, allowing the researchers to explore existing services, policies, and local knowledge from the perspective of the caregivers who were accessing services.

Participants: A total of eight participants $(N=8)$ met the criteria for participation. Six of the participants in the study were adoptive parents and two were foster parents. Of the eight participants, seven were female and one was male. Three of the study participants resided in rural locations and five in urban centers.

Findings: Results indicated that caregivers pass through four stages of caregiving, with each stage having a distinct primary need and requiring supports and services that best address that need. Three additional themes related to service delivery also emerged: caregiver considerations, program factors, and building on strengths.

Conclusions: Study results emphasize the need for further research exploring the concept that caregivers experience specific caregiving stages, and that programming factors should align with caregivers' needs in each of these stages. Participants' narratives also suggest that the results of this study may be relevant beyond its regional specificity and limited sample size, and may in fact reflect the experiences of caregivers across jurisdictions.
\end{abstract}

Prenatal alcohol exposure can lead to a variety of cognitive, behavioural and neurological deficits, including permanent structural damage to the brain, all of which fall under the umbrella term of Fetal Alcohol Spectrum Disorder (FASD) (Chudley et al., 2005). Often, the most severe problems that individuals with FASD experience are deficits in areas important to independent functioning, such as memory, attention, abstract thinking, judgment, and cause-and-effect reasoning (Rasmussen, McAudley, \& Andrew, 2007). These deficits are the result of central nervous system dysfunction and are referred to as primary disabilities. Secondary disabilities, such as trouble with the law, disrupted school experience, and drug and alcohol abuse, to name a few, are consequences of primary disabilities. Secondary disabilities, also described as adverse outcomes, are often exacerbated by "limited understanding (and availability) of effective interventions" (Rasmussen, Andrew, Zwaigenbaum, \& Tough, 2008). Consequently, individuals with FASD face challenges in obtaining and maintaining employment, maintaining social relationships, and applying adaptive functioning skills (Jirikowic, Kartin, \& Olson, 2008).

Hodges, Ferreira, Israel, and Mazza (2007) suggested that systems of care be defined as "broad flexible array[s] of services and supports for a defined population” (p. 9). Health, education, mental health, and respite services are examples of the supports that make up systems of care for individuals with FASD. Due to the complexity of the problems associated with FASD, multiple services across a variety of systems of care are required. Although the types of services required vary with each individual's needs or life stage, many caregivers and individuals with FASD report a similar set of barriers to accessing services. For example, problems with policy, funding, and eligibility structures have been documented (Rutman \& Van Bibber, 2010). 
Approximately $\$ 5.3$ billion Canadian dollars annually are spent on services for individuals with FASD (Stade et al., 2009), yet even so, these systems of care may not always meet the needs of individuals with FASD. When an individual with FASD has unmet or unrecognized needs, the consequences for the individual and their family—such as placement disruption, or inappropriate or absent interventions that lead to poor outcomes-can be devastating (Olson, Oti, Gelo, \& Beck, 2009).

Caregivers raising alcohol-affected children must juggle multiple roles, such as parent, advocate, teacher, and therapist (Brown, Sigvaldason, \& Bednar, 2005). Streissguth et al. (2004) documented the importance of positive and stable family environments to the outcomes of those with FASD, and Brown et al. (2005) have documented the link between a caregiver's belief in their ability to care for an individual with FASD and the caregiver's ability to cope with daily challenges. Given these findings, it seems important to understand how the systems of care designed to meet the needs of individuals and families impacted by FASD are perceived by the caregivers who try to use them. However, caregivers' perceptions of their interactions with established systems of care have yet to be examined.

\section{Methods}

\section{Study Objectives}

The goals of this study were to (1) gain improved understanding of the needs of caregivers of individuals with FASD, (2) gain improved understanding of the experiences of these caregivers as they attempt to access and implement supports in their daily functioning, and (3) gain improved understanding of their perceptions of the strengths and challenges of the systems of care that are in place for individuals with FASD.

\section{Sample and Participant Selection}

This was a qualitative study informed by grounded theory. This choice of theory allowed for documentation of rich descriptions that captured the perspectives and voices of caregivers as they shared their experiences of interacting with systems of care designed to minimize the impact of FASD-related issues. Participants for this project were chosen with the support of recruiters from a social service agency in Edmonton, Alberta. A presentation introducing the study was made by the authors to agency staff, who then distributed a study poster to caregivers enrolled in agency programs. Interested caregivers contacted researchers and were screened to ensure that they met the criteria: each participant had to be the person taking primary responsibility for care, either currently or within the past three years, of one or more individual(s) diagnosed with FASD. Eight candidates $(N=8)$ contacted the authors, and all eight met the criteria for participation.

Six participants were adoptive parents and two participants were foster parents. The foster parents were a married couple, with three individuals with FASD, from 17 to 25 years of age, residing in their home at the time of the interview. Of the six adoptive parents, three were parenting youths between the ages of 13 and 18 years. One adoptive parent was caregiving for two children with FASD, both under the age of 10 years. Two participants were the legal guardians of adult children. Of the eight participants, seven were female and one was male. All were between 40 and 60 years of age. Three resided in rural locations and five in urban centers. The relationship between the study participants was not known. Prior to the interviews, all participants were provided with an oral explanation of the research process, and consent forms were signed securing permission for audio taping and transcription.

\section{Qualitative Methods}

Information for this study was gathered using an in-depth interviewing method, allowing the researchers to explore existing services, policies, and local knowledge from the perspective of the individuals accessing these services, rather than from the organizations' or governmental agencies' statements of their service goals. Questions focused on the experiences that caregivers had had with FASD service providers, exploring themes such as the type of support required, the type of services utilized, the perceived benefit of support services, and the areas where services were perceived to require improvement. Interviews ranging in duration from 60 to 120 minutes were conducted in person at locations convenient to the participants. Interviews were then transcribed and the transcriptions reviewed with study participants for accuracy.

Researchers analyzed the transcripts using a two-stage coding process suggested by Charmaz (2006) that followed a modified grounded-theory approach. The preliminary coding analysis involved a review of the transcripts in a line-by-line format. Data then underwent a second coding process which arranged the information into emergent themes that represented larger sections of data.

\section{Results}

In this study, eight individuals were interviewed who were caregiving for persons diagnosed with FASD. Although each narrative differed in its specific circumstances-such as the participants' location (urban versus rural), socioeconomic class, education level, or the degree of deficits and developmental levels of the loved ones with FASD - shared experiences emerged across all eight interviews. In particular, three main themes related to service delivery were noted (1) caregiver considerations (Table 1), (2) program delivery challenges, and (3) building on strengths.

\section{Caregiver Considerations}

Upon analysis of the completed interviews, it became clear that participants had each experienced similar processes in their roles as caregivers. Four key stages were identified seeking, reflecting, collaborating, and planning. These 
stages were not necessarily linear or dependent on the age of the individual being cared for; rather, they reflected the different needs of caregivers at different times.

Seeking. In this stage, caregivers work to better understand the individual for whom they are caring. Participants reported that problems with their children with FASD were evident prior to the individuals' beginning school, and become more pronounced and complex with age. The initial caregiving years (when the affected individuals were approximately 2-10 years of age) were spent trying to identify specific problems and gather information about supports and services.

During this stage, caregivers reported spending large amounts of time gathering and organizing masses of information as to what services were available, to the point where many of them felt overwhelmed and depleted. One caregiver reported, "I feel like I eat, sleep, and breathe FASD." This emotional state appears to be the impetus for the primary features described in stage two of the process.

Reflecting. In this stage, caregivers become more reflective about their own caregiving practices; in particular, they reported feeling inadequate in their caregiving roles. Specifically, caregivers described feelings of self-doubt and blame for the problems their children were experiencing. A common perception among study participants was that family members, schools, and service providers often attributed difficult behavioral issues to perceived caregiving deficits. One caregiver stated:

I haven't attended any family functions in the past two years because I am afraid of what [my son] might do ... I don't think I could handle my family talking about me ... I know what they think of me... I am a bad parent.

She, like others in this stage, reported feeling "too tired" to keep working to change her family members' perceptions, resulting in her withdrawal from friends, family, and social supports.

Collaborating. During this stage, caregivers described a convergence between understanding their role as caregivers and understanding the needs of their loved ones with FASD. They reported that this led to a renewal of their efforts to invest in the support-seeking process; for example, they began adjusting their family and employment routines in order to make time for advocacy and support seeking. One caregiver reported that after an extended period of solitude and reflection, she realized she was going to have to create the solutions that would work for her family: "My children need me . . . if I don’t do it, who will?”

At this stage, caregivers described making changes to accommodate the cost and time commitments required to provide their child with an adequate level of specialized support. One caregiver stated, "We had to decide what our priorities would be; the priority is the children . . . there is nothing left for anything extra." Another caregiver described the process of finding services as "a full-time job." Another mentioned the large out-of-pocket expenses for services such as ongoing psychotherapy.

Planning. Caregivers described stage four as a time of reconciliation; they no longer felt they were simply responding to the needs of their loved one, and they were now able to focus on the strengths and contributions their loved one brought to the family and begin planning for the future. One caregiver indicated that although she had initially struggled to accept her child's diagnosis with FASD and its implications, she was eventually able to find acceptance and move forward: "I have spent a lot [of] time working through my grief with a counsellor; this has been a really important part of moving forward for me." Another caregiver stated:

My husband and I never expected this to be our life when we adopted, but it is ... We love our children; we wouldn't change a thing . . . So we have to do what is best for [our children] because, after all, they didn't ask to be born with FASD.

\section{Program Delivery Challenges}

As well as identifying specific stages of caregiving, the study identified a number of challenges in existing program delivery. The need for increased accessibility, extension of services to all family members, provision of services across the lifespan, flexibility around funding access and application, and the need for the provision of legal supports were the main program challenges described by caregivers.

One of the challenges that participants reported was enduring long waitlists for FASD services, such as assessment, life skills programming, social skills training, and parenting support services. One caregiver said that her child had been on a waitlist for over 18 months, and that by the time the call arrived, the child was no longer eligible for the service, due to the age restrictions of the program. Another caregiver reported that she had stopped accessing services because the staff turnover rate was so high, the child never had time to establish relationships with staff members. Participants also identified a need for support in completing the paperwork necessary to enable program participation. As one caregiver explained, "I needed the service because I am at my wits' end; I don't have the energy left to jump through the hoops needed to get the support."

Extending the scope of service to include families as a whole was identified as a need. One participant suggested that it would help if her husband had other men to talk to about the difficulties with their son: "My husband had different dreams for our son than I did ... I think that our son having FASD hasn't really sunk in for him yet the way it has with me, but there isn't anyone for him to talk to." Another caregiver mentioned the need for individual counselling for the siblings of her son with FASD, stating that "funding is not available for anyone other than [my son]". 
Table 1

An Overview of the Four Stages of Caregiving with the Required Services and Supports

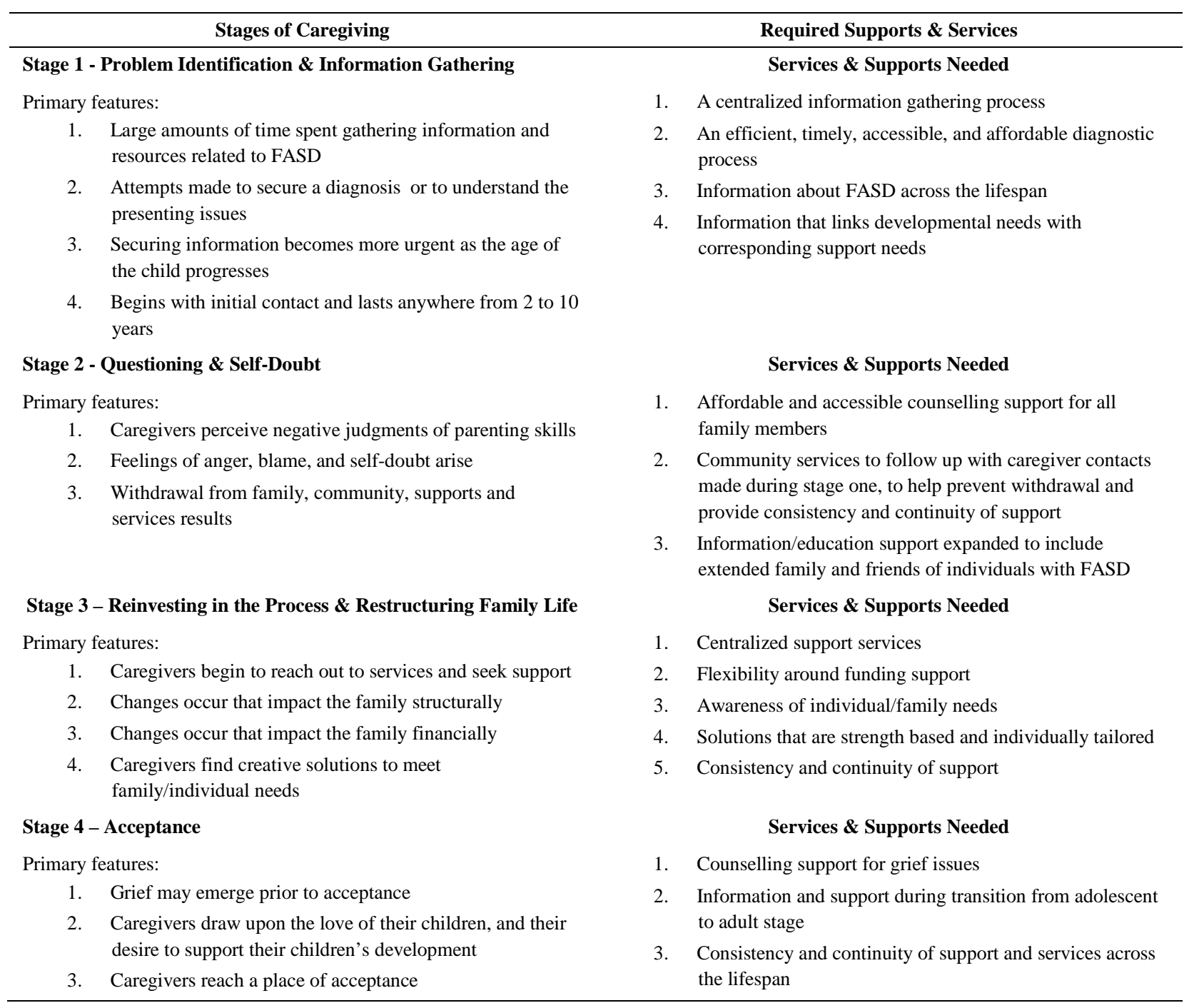

Most participants were able to identify specific services dedicated to young children with FASD, but noted that services declined in availability as children aged. Only one provider of mentoring services was identified as able to work with adolescents with FASD. Participants were unable to identify FASD programming for adults, positing that monetary support was the main adult support. One caregiver noted, "The only services available for my son [age 17] are the police, jail, and the emergency room.”

Many of the participants expressed anxiety about their lack of legal authority over their children past the age of eighteen, saying that their children were not capable of managing the day-to-day requirements of life without continued parental involvement. During one of the interviews, a caregiver stated, "Unless she agrees to sign over control, we are powerless.”
All study participants expressed concerns related to funding structures and restrictive funding guidelines. For instance, many discussed the limited funding for respite care, with one caregiver asking, "Would you look after such highneeds kids for minimum wage?” Linked to this issue were difficulties finding qualified and knowledgeable individuals to provide respite services. One caregiver added that children with FASD do not transition well, making it necessary to introduce a respite worker into the family over a number of visits, which is time that is not currently recognized or funded by existing systems of care.

Many caregivers mentioned that they were aware of available funding established to help alleviate the financial burden associated with FASD, but expressed frustration that the money could not be used for what they perceived as "real" needs. One family related that they needed to 
drive over 5,000 kilometers a month to bring their daughter to and from appointments, yet they were unable to get funding to help cover gas expenses.

\section{Building on Strengths}

Participants agreed that there are strengths in existing services, and that to build on these strengths, an increase in the centralization of services is desirable. In particular, facilities that could house multiple service providers trained to deal with a range of issues related to FASD, thus allowing for "one-stop shopping," are needed. Participants believed the benefits of such facilities would include the elimination of duplication of services, multiple phone calls, multiple recitations of a family's FASD story, minimization of paperwork, reduced travel and time costs, and the creation of a place where caregivers and individuals affected by FASD could connect and make contact with one another. Rural study participants added that it would be beneficial if the centralized services provided mobile teams for those individuals outside of the urban centers.

\section{Discussion}

Bronfenbrenner (1994) stated that ecological models of human development are concerned with the processes and conditions of human beings as they unfold in the actual lived environments of individuals across the lifespan. In keeping with this model, researchers in this study attempted to view existing interventions through an ecological lens in order to gain a better understanding of the experiences, needs, and perceptions of caregivers attempting to access and implement supports in daily functioning. An additional goal of this project was to gain a better understanding of the strengths of existing services and supports and the challenges in accessing them. Study results identified four stages of caregiving and showed that caregiver needs changed across these stages. Because of these changes, caregivers articulated that systems of care must be flexible and responsive, in order to ensure the best fit in support delivery.

Table 1 depicts the supports and services that best match the primary features of each caregiving stage. Participants suggested that collaboration between caregivers and service providers would foster the emergence of support structures that are more intentional about seeking information and implementing results, and better at matching programs to the specific needs of caregivers in each of the four stages.

Research into program delivery challenges has begun to be documented across a variety of jurisdictions in Canada (Fuchs, Burnside, \& Marchenski, 2010; Rutman \& Van Bidder, 2010). Participants in this study highlighted program challenges similar to those identified in other studies, such as reduced program access resulting from long waitlists, frequent staff turnover, and large amounts of paperwork, as well as the need for flexibility around access and use of funds. When compiled with other research findings, results suggested that service needs may not be regionally dependent, but instead reflect the challenges of the disability in general. Participants' narratives point to the need for services that are more intentional about the alignment of support with caregiver needs, for more centralized and coordinated services, and for increased accessibility and flexibility around funding and program participation requirements; they also highlighted the need for an expansion of services to meet the requirements of individuals with FASD across the lifespan. Caregivers in the study expressed optimism that current services can build on existing strengths as they work towards these goals.

\section{Limitations of the Present Study}

Although the small sample size and geographical specificity limit the generalizability of the results of this study, research examining the caregiving needs of foster parents caring for individuals with FASD in other jurisdictions (Fuchs, Burnside, \& Marchenski, 2010; Rutman \& Van Bidder, 2010) has produced similar results, allowing the authors to hypothesize that the results of this study may reflect the experiences of caregivers across jurisdictions, and highlighting the importance of expanding this project to other geographical locations. Lastly, study results also emphasize the need for further research exploring the relationship between caregiving stages and the type, alignment, and timing of services, to assist in increasing positive outcomes for individuals and families impacted by FASD.

\section{References}

Bronfenbrenner, U. (1994). Ecological models of human development. In P. Peterson, E. Baker, \& B. McGraw (Eds.), International encyclopedia of education ( $2^{\text {nd }}$ ed., Vol. 3). Oxford, United Kingdom: Elsevier.

Brown, J., Sigvaldason, N., \& Bednar, L. (2005). Foster parent perceptions of placement needs for children with fetal alcohol spectrum disorder. Children and Youth Services Review, 27, 309-327.

Charmaz, K. (2006). Constructing grounded theory: A practical guide through qualitative analysis. Thousand Oaks, CA, United States: Sage Publications, Ltd.

Chudley, A., Conry, J., Cook, J., Loock, C., Rosales, T., \& LeBlanc, N. (2005). Fetal alcohol spectrum disorder: Canadian guidelines for diagnosis. Canadian Medical Association Journal, 172, 1-17.

Fuchs, D., Burnside, L., \& Marchenski, S. (2010). Children with FASD-related disabilities receiving services from child welfare agencies in Manitoba. International Journal of Mental Health and Addiction, 8, 232-244.

Hodges, S., Ferreira, H., Israel, N., \& Mazza, J. (2007). Lessons from successful systems (Issue Brief No. 1). Tampa, FL, United States: University of South Florida, Louis de la Parte Florida Mental Health Institute, Research and Training Center for Children's Mental Health.

Jirikowic, T., Kartin, D., \& Olsen, H. (2008). Children with fetal alcohol spectrum disorders: A descriptive 
profile of adaptive function. Canadian Journal of Occupational Therapy, 75, 238-248.

Olson, H. C., Oti, R., Gelo, J., \& Beck, S. (2009). "Family matters": Fetal alcohol spectrum disorders and the family. Developmental Disabilities Research Reviews, 15, 235-249.

Rasmussen, C., Andrew, G., Zwaigenbaum, L., \& Tough, S. (2008). Neurobehavioural outcomes of children with fetal alcohol spectrum disorders: A Canadian perspective. Paediatrics \& Child Health, 13, 185191.

Rasmussen, C., McAuley, R., \& Andrew, G. (2007). Parental ratings of children with Fetal Alcohol Spectrum Disorder on the Behavior Rating Inventory of Executive Functioning (BRIEF). Journal of Fetal Alcohol Spectrum Disorder, 5, 1-8.

Rutman, D. \& Van Bibber, M. (2010). Parenting with Fetal Alcohol Spectrum Disorder. International Journal of Mental Health and Addiction, 8, 351-361.

Stade, B., Ali, A., Bennett, D., Campbell, D., Johnston, M., Lens, C., Tran, S., \& Koren, G. (2009). The burden of prenatal exposure to alcohol: Revised measurement of cost. Canadian Journal of Clinical Pharmacology, 16, 91-102.

Streissguth, A., Bookstein, F., Barr, H., Sampson, P., O’Malley, K., \& Young, J. (2004). Risk factors for adverse life outcomes in fetal alcohol syndrome and fetal alcohol effects. Developmental Behavioural Paediatrics, 25, 228-238. 